\title{
WIRELESS DISTRIBUTED ACQUISITION SYSTEM FOR NEAR INFRARED SPECTROSCOPY - WDA-NIRS
}

\author{
J. SAFAIE, ${ }^{*}$, R. GREBE*, H. ABRISHAMI MOGHADDAM ${ }^{*, *}$ \\ and F. WALLOIS ${ }^{*}, \dagger$ \\ *GRAMFC - Inserm U1105 \\ UFR of Medicine of University of Picardie Jules Verne \\ 80036 Amiens, France \\ ${ }^{\dagger}$ GRAMFC - Inserm U1105 \\ EFSN Pediatrique, North Hospital \\ 80056 Amiens, France \\ $\star$ Faculty of Electrical Engineering \\ K. N. Toosi University of Technology \\ 16315-1355, Tehran, Iran \\ §javad.safaie@u-picardie.fr
}

Received 2 April 2013

Accepted 6 May 2013

Published 18 June 2013

\begin{abstract}
The wireless distributed acquisition system for near infrared spectroscopy (WDA-NIRS) is a portable, ultra-compact, continuous wave (CW) NIRS system. Its main advantage is that it allows continuous synchronized multi-site hemodynamic monitoring. The WDA-NIRS system calculates online changes in hemoglobin concentration based on modified Beer-Lambert law and the tissue oxygenation index based on the spatial-resolved spectroscopy method. It consists of up to seven signal acquisition units, sufficiently small to be easily attached to any part of the body. These units are remotely synchronized by a PC base station for independent acquisition of NIRS signals. Each acquisition module can be freely adapted to individual requirements such as local skin properties and the microcirculation of interest, e.g., different muscles, brain, skin, etc. For this purpose, the light emitted by each LED can be individually, interactively or automatically adjusted to local needs. Furthermore, the user can freely create an emitter time-multiplexing protocol and choose the detector sensitivity most suitable to a particular situation. The potential diagnostic value of this advanced device is demonstrated by three typical applications.
\end{abstract}

Keywords: NIRS; optical spectroscopy; body area network; microcirculation.

This is an Open Access article published by World Scientific Publishing Company. It is distributed under the terms of the Creative Commons Attribution 3.0 (CC-BY) License. Further distribution of this work is permitted, provided the original work is properly cited. 


\section{Introduction}

Jöbsis was one of the first to show that the near infrared (NIR) range of the light spectrum (650$1000 \mathrm{~nm}$ ) can be used to monitor certain physiological phenomena. ${ }^{1}$ Near infrared spectroscopy (NIRS) is now an innovative clinical method to assess information concerning local hemodynamic and metabolism in the brain and muscles. ${ }^{2-7}$ Three types of NIRS instruments have been proposed according to the light source: time-resolving, frequency domain and continuous wave (CW) machines. The simplest and most widely used machines at the present time use continuous wave light sources (CW system). This kind of instrument measures attenuation of light of different wavelengths and can therefore be used to determine changes in chromophore concentrations such as oxygenated $(\mathrm{HbO})$ and deoxygenated $(\mathrm{HbR})$ hemoglobin, induced by local changes of microcirculation and/or metabolism.

However, light absorption measurements alone cannot be used to determine absolute concentrations of chromophores; information about the photon path length is therefore necessary, but is not provided by the $\mathrm{CW}$ method. Absolute values can be estimated within certain limits by using multiwavelength systems or spatial-resolved spectroscopy (SRS). ${ }^{8,9}$ The advantages of CW NIRS instruments are their relatively low price and small size; they can even be designed as portable clinical devices.

Most of the commercially available NIRS instruments are relatively expensive, cumbersome, heavy and inflexible in terms of their applications, mainly due to the use of glass fibers for light transfer and photomultiplier tubes (PMTs) for photon detection. More recent developments now use lightemitting diodes (LEDs) as light sources and silicon photo-diodes (SPDs) as detectors, ${ }^{10,11}$ which drastically lowers the price, size and complexity of these instruments. LEDs offer numerous advantages compared to laser diodes (LDs): LEDs can be positioned directly on the skin, making optical fibers obsolete, they are inexpensive and generally not harmful for the patient's eyes, which is very important in the clinical setting. ${ }^{10}$

Moreover, the light power-current relationship of LDs is linear for relatively high injection currents only. In the low-current mode, required for short emitter-detector distances (EDDs), there is a threshold, resulting in marked nonlinearity. In contrast, LEDs have a linear optical power-current relationship, allowing to drive them also by very low currents. The replacement of PMTs or avalanche photo diodes by SPDs has the advantage of avoiding the bias of the high voltages required by former, while providing sufficient sensitivity. In addition, there is no need to use optical fibers, as SPDs can be applied directly to the skin.

Several teams have tried to develop more flexible instruments suitable for brain monitoring. Yurtsever et al. developed a portable NIRS system based on a pocket $\mathrm{PC}$ as controller and processor unit. They chose LEDs with wavelengths of 730 and $850 \mathrm{~nm} .{ }^{12}$ However, probably due to technical limitations at that time, they were unable to use a pocket $\mathrm{PC}$ as main station and monitoring system.

Nevertheless, the idea of combining a controller unit and a base station $\mathrm{PC}$ in a new powerful PDA or pocket $\mathrm{PC}$ remains a fairly interesting solution. Muehlemann et al. proposed a wireless miniaturized in vivo NIR imaging system using LEDs with wavelengths of 760 and $870 \mathrm{~nm}$ and SPD. To achieve greater flexibility concerning the patch structure, they used flexible boards. ${ }^{10}$ Zhang et al. developed a portable NIRS system based on an embedded system board that can operate autonomously, but it has the disadvantage of requiring a high-capacity battery as power supply, making it large and heavy. ${ }^{13}$

In this paper, we present a new device termed wireless distributed acquisition NIRS (WDA-NIRS). Based on advanced digital circuit design technology, it provides considerable progress in terms of miniaturization and portability, while offering:

- Important advancements concerning flexibility in LED-detector configuration and modes of operation.

- Simultaneous signal acquisition from up to seven body positions (even during movement) by independent low-power battery-operated distributed acquisition modules.

- High dynamic input range of each module via individual programmable drivers for each light source and programmable gain amplifiers (PGA) for each detector.

- Software-driven auto-calibration and adaptive control of light pulse generation and signal acquisition allowing adaptation to different experimental and environmental conditions.

- High signal-to-noise ratio (SNR) based on individual alignment of each light source. 
The following studies were conducted to demonstrate the performance and highlight the new features of WDA-NIRS:

- Validation with a sensitivity mapping phantom using a home-made liquid phantom was previously presented in Ref. 14 .

- Evaluation of our device with NIRO-200NX (cerebral oxygenation measuring system, Hamamatsu ${ }^{\circledR}$ ) by arterial occlusion experiments on human arm.

- Breath-holding experiments to demonstrate simultaneous acquisition of multiple NIRS signals from different sites, each providing information from different tissue depths.

- Stair negotiation experiments to demonstrate the applicability of WDA-NIRS for portable distributed signal acquisition even in freely moving subjects.

\section{Methodology}

\subsection{Instrumentation}

The WDA-NIRS device presented here was designed by applying a field programmable gate array (FPGA) as central controller/processor. The whole system consists of up to seven distributed modules (each module includes a patch, a control and processing unit, and a wireless communication unit), and a PC-based graphical user interface (GUI) (see Fig. 1).

\subsubsection{Patch}

The patch constitutes the data acquisition interface between the system and the subject (patient body or phantom) comprising an appropriate geometry of light sources and detectors. Therefore, it plays a key role in reliable signal acquisition.

The current system supports up to 16 light sources ( $8 \mathrm{red} / \mathrm{IR}$ pairs) and 2 detectors that can be extended up to 8 detectors. For the experiments described in this paper, two types of patches were designed and produced. The first patch [see Fig. 1(d)] is similar to the widely used commercial patches and contains one detector and four emitter pairs. This design is commonly used to investigate tissues at relatively well-known distinct locations at a certain depth. This kind of NIRS signal acquisition has the inherent disadvantage that the acquired signal contains not only the desired information from the region of interest, but also from all layers crossed by the photons from the skin to the deepest tissue layer reached and from the return path.

The second patch [see Fig. 1(c)] was designed in order to provide a hardware basis to overcome this

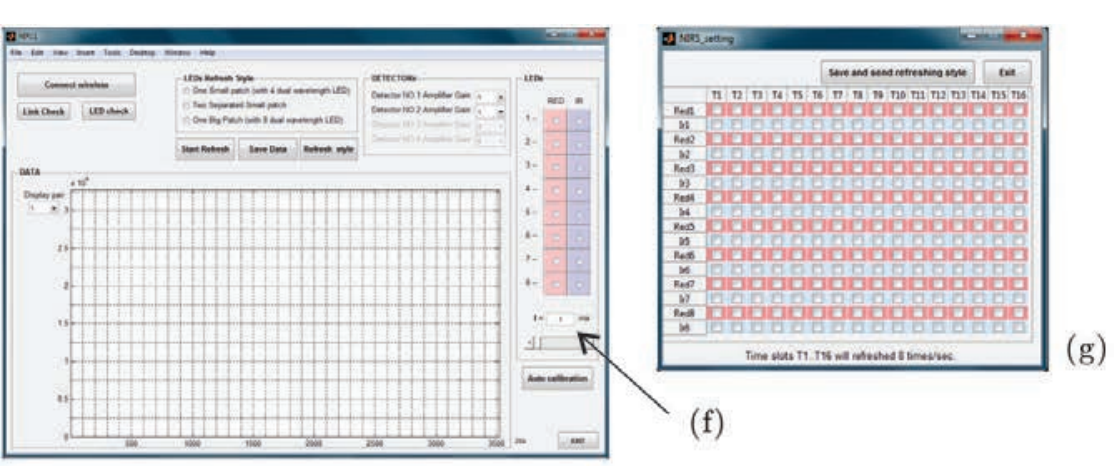

(e)
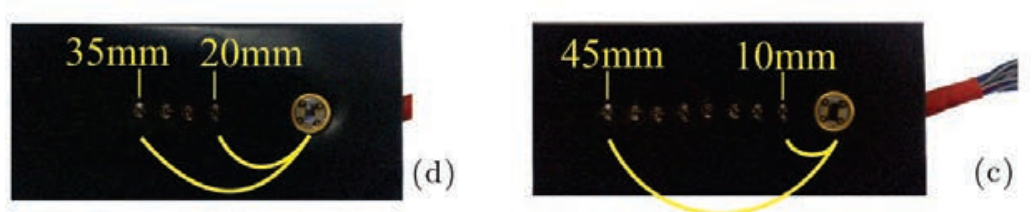

(a)

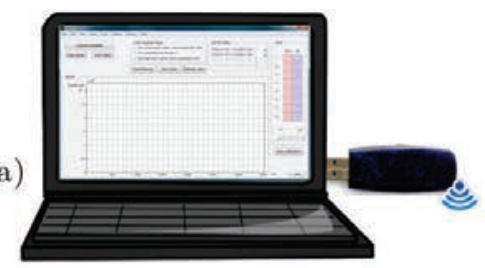

(g)

(b)

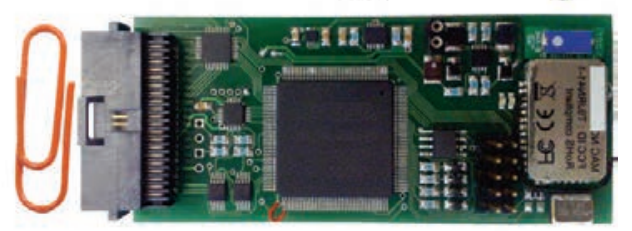

Fig. 1. WDA-NIRS system: (a) base station, (b) control and processing unit $\left(35 \times 80 \times 9 \mathrm{~mm}^{3}\right)$, (c) high-resolution patch $\left(30 \times 70 \times 10 \mathrm{~mm}^{3}\right)$, (d) patch with standard configuration $\left(30 \times 70 \times 10 \mathrm{~mm}^{3}\right)$, (e) main GUI window, (f) interface for individual current adjustment (light output) for each LED, (g) interface for emitter time-domain multiplexing programming, allows the user to define arbitrary refreshing patterns adapted to actual patch configuration, number of channels, dark references, etc. 
disadvantage. The number of emitters was therefore increased to acquire signals containing progressively additional information from deeper tissue layers by stepwise increasing the EDD. ${ }^{14,15}$ The availability of this type of multi-distance signal is the prerequisite for application of decomposition algorithms as already proposed by Zhang et al. ${ }^{16}$ Special efforts have to be taken to allow acquisition of signals from such a wide range of distances in a single measurement, as the light intensity necessary for a distance of $40 \mathrm{~mm}$ is typically about 1000 times higher than that necessary for a distance of $10 \mathrm{~mm}$ to achieve a sufficiently high SNR for all signals. To meet this requirement, each light source in our device is independently programmed and/or automatically optimized online.

There are essentially two different types of components available to generate NIR light pulses; LEDs and LDs. In this device we preferred LEDs. By applying the same maximum permissible exposure (MPE) criterion for the skin, more NIR photons (per time and area unit) can be injected into the tissue since LEDs create lower power density than equivalent LDs. ${ }^{17}$ In addition, laser light sources undergo considerable heating of their semiconductor junctions, preventing from positioning them directly on the skin. Optical fibers must therefore be introduced to transfer the light to the tissue, while LEDs can be applied directly on the skin. ${ }^{10}$ Nevertheless, the potential hazard of NIR light emitted by LEDs for patient and personnel eyes must also be considered, as described in Ref. 18.

In the NIR range of the spectrum, photons are mainly scattered in tissues with very low wavelength dependency. In contrast, absorption is highly wavelength dependent and specific for the various absorbers. The lower boundary of the optical window $(\approx 650 \mathrm{~nm})$ is determined by the high absorption factor for $\mathrm{HbR}$, and its upper boundary $(\approx 975 \mathrm{~nm})$ is determined by the absorption factor for water. Around $800 \mathrm{~nm}$ (isobestic point), the molar extinction coefficient is the same for $\mathrm{HbO}$ and HbR. To ensure unequivocal differentiation between changes in $\mathrm{HbO}$ and $\mathrm{HbR}$ concentrations and to achieve a sufficiently high SNR, at least two sufficiently different wavelengths in the NIR band around $800 \mathrm{~nm}$ are usually applied. Strangman et $a .^{19}$ and Uludag et $a l .{ }^{20}$ conducted a detailed analysis of the most appropriate wavelength.
Following their results and taking into account the commercial availability of components, we selected a dual-wavelength LED with 760 and $850 \mathrm{~nm}$ wavelengths housed in a $3 \mathrm{~mm}$ package (L760/850 Epitex ${ }^{\circledR}$, Inc). This LED radiates light with a power of up to $17 \mathrm{~mW}$ with a very good linearity between driving current and emitted light intensity from very low to high current for short to long EDDs, respectively.

The detector consists of a high-sensitivity monolithic SPD with integrated amplifier (ODA6WB-500M, Optodiode ${ }^{\circledR}$ Co.) and a large active area $\left(6 \mathrm{~mm}^{2}\right)$. The high-gain trans-impedance amplifier integrated in this SPD dramatically reduces the output noise. Applying a $500 \mathrm{M} \Omega$ resistor in the feedback path of this amplifier results in a light sensitivity higher than $250 \mathrm{~V} / \mu \mathrm{W}$. In the version of the system presented here, the maximal overall sampling rate is 8 samples/s for each wavelength of each LED, if the user activates all of the 16 light sources. Accordingly, for one channel (one LED-SPD), a sampling rate of 64 samples/s would be possible.

The housing of the emitters and detectors (optodes) plays an important role in NIRS systems, as the quality of the contact between optodes and the skin directly affects the quality of the acquired signals. To achieve good contact, the LED/SPD holder must be made of relatively soft and flexible material with a sufficiently hard structure to keep the LEDs/SPDs in place, offering a certain surface adhesiveness and preventing any ambient light from entering the detectors. Liquid polymerizable silicon (Elastosil, Wacker Silicones ${ }^{\circledR}$ ) was found to be the most appropriate solution for this purpose.

Patches were manufactured in three steps: firstly, a relatively thin silicon layer was spread out into a mould. After drying, optodes were then positioned and all connections were wire-wrapped to a flat cable. Finally, the assembly was covered with a second silicon layer, which fused with the first layer and therefore enclosed all electronic components and wires in a flat rectangular silicon cuboid structure.

Application of the patch on the skin showed that it was very well adapted to the local surface geometry (curvature) and remained in place and shape with only moderate pressure from an elastic tubular net bandage (Asterie ${ }^{\circledR}$ ), due to the intrinsic adhesiveness between the silicon surface and the skin. 
The $10 \mathrm{~mm}$ thick silicon patch is also a perfect light insulator, which is important to reduce the effect of extraneous light. This can be easily verified by a simple "all LEDs off" test at any time during measurement. Time-division multiplexing (TDM) programming allows the user to integrate background light measurements, whenever necessary during an experiment. If desired, the patch can also be easily covered by light-insulating material (such as a black fabric), as it does not comprise any optical fibers or other interfering structures.

\subsubsection{Control and processing unit}

This unit executes all controlling and processing tasks, such as creating individual TDM protocols, auto-calibrating light emitters, adjusting PGAs, performing arbitrary processing tasks, etc. Careful design of this unit is essential to achieve the required specifications and improve the performance of the measurement instrument. This is why we have chosen a FPGA as central processing unit, as it provides the following advantages:

- It combines all digital hardware in a low power consumption chip, which is essential for a portable battery-operated system.
- It allows extended real-time signal processing; its high speed and parallel structure provide an ideal basis for implementation of algorithms for signal acquisition, conditioning and expanded online preprocessing for special clinical applications.

- It provides flexibility in terms of the required LED-detector configuration and modes of operation. Light pulse generation and signal acquisition control can be easily adapted to various kinds of patches by simple software upgrading.

A detailed block diagram of the system hardware is presented in Fig. 2. The main functions of this part are to:

- Establish a wireless link to the PC to receive instructions and send data. The wireless link uses a class 1 Bluetooth transceiver ("RN-41", Rovingnetworks $\left.{ }^{\circledR}\right)$. It supports the related electromagnetic compatibility (EMC) standards. Other devices using the industrial scientific medical (ISM) band do not interfere with the wireless transmission, due to built-in error correction and frequency hopping technologies. ${ }^{10}$ In addition, no disturbance of medical equipment by Bluetooth or vice versa has been reported to date. ${ }^{21}$ We have also verified that the Bluetooth device has no

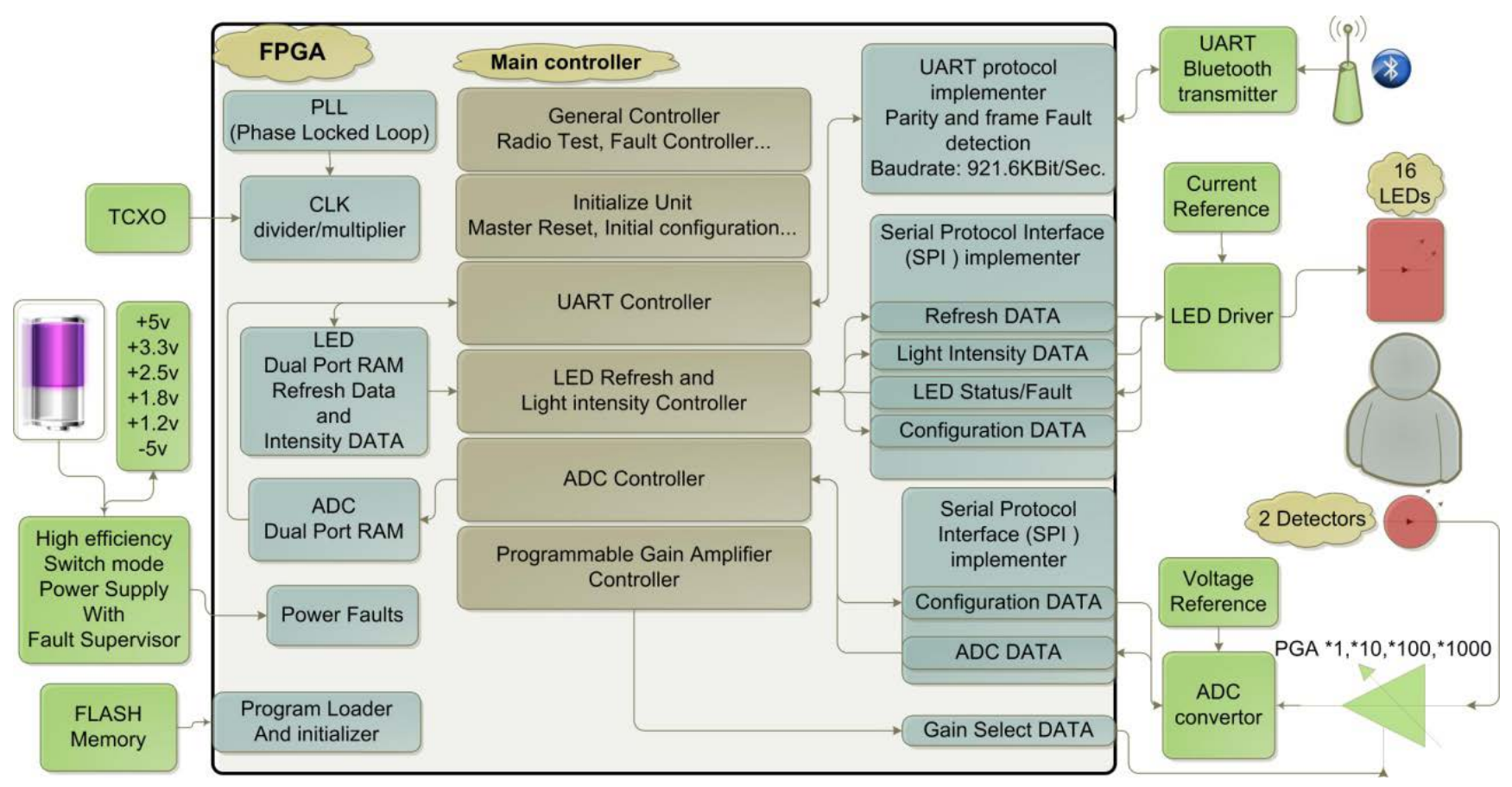

Fig. 2. Hardware block diagram of the WDA-NIRS: The FPGA is the main component determining all of the various input/ output units in parallel. 


\section{J. Safaie et al.}

influence on the acquired signals by performing identical experiments with wire and wireless UART that provided identical experimental results. The FPGA contains a high-speed UART protocol implementer to drive the Bluetooth transceiver and to control data throughput and error handling. It also answers $\mathrm{PC}$ radio link check requests. In the event of loss of the radio link, an alarm is generated on both sides (PC and control and processing unit).

- Drive programmable current sources and prepare auto-calibration. This part (TLC5923, Texas Instruments $\left.{ }^{\circledR}\right)$ contains 16 programmable constant current sources, which can each be programmed independently in 128 levels. The user (or the system automatically) can adjust the emitted light individually for each LED according to the experimental conditions such as EDD, local skin color, etc. This feature extends the measurement depth-range, while preserving the required SNR.

- Implement different types of TDM according to predefined standard refreshing patterns. A special look-up table is designed inside the FPGA via a dual port RAM, storing information concerning various refreshing schedules. These predesigned refreshing patterns correspond to previously designed patches; an example is presented in Fig. 1. They can be selected and manipulated via a MATLAB GUI. The FPGA also contains a serial protocol interface (SPI) unit for communication with the LED driver unit to control and handle error conditions such as broken LED or overheating of the LED driver. In addition, the user can define an arbitrary pattern to adapt to the needs of special experimental conditions, e.g., by adding a dark state in the refreshing routine (all light sources "off"), the environmental light can be measured and registered continuously to compensate the effect of this light by online (or offline) processing.

- Acquire and condition analog signals by the high-precision PGA (AD8253, Analog Devices ${ }^{\circledR}$ ), and convert them into digital form with the 16 bit, $500 \mathrm{kHz}$ analog to digital converter (ADC) (AD7699, Analog Devices ${ }^{\circledR}$ ). The gain of the PGA can be adjusted to four levels: $\times 1, \times 10, \times 1$ 00 or $\times 1000$; the dynamic range of the input signal for the ADC can therefore be completely covered by using this PGA.
- Provide the various voltage sources required from a single $3.6 \mathrm{~V}-1000 \mathrm{mAH}$ Li-ion rechargeable battery using a high-efficiency switch-mode power supply. Three kinds of switch-mode power supply (buck, boost and inverting) are used, resulting in reduction of total power consumption. Switching frequencies have been chosen much higher than the highest frequency of the signal of interest to minimize noise due to the power supply. Additional techniques including decoupling power supplies and shielding planes have been applied to improve the overall SNR.

\subsubsection{Wireless communication unit}

This unit consists of two class 1 Bluetooth component, one integrated in the control and processing unit, and the other integrated in a USB key connected to the PC. It provides data transfer with a rate of up to $921.6 \mathrm{Kbits} / \mathrm{s}$ over a distance of up to $100 \mathrm{~m}$. The PC can simultaneously support up to seven channels, corresponding to seven independent acquisition modules (including a patch, a control and processing and a wireless unit) attached separately at arbitrary sites on the patient's body. Standard protocols for error detection and retrieving missed data are integrated into these components to ensure error-free data transfer.

\subsubsection{PC-based GUI}

A user-friendly MATLAB ${ }^{\circledR}$ GUI allows easy (re) configuration of the WDA-NIRS system as well as data acquisition via the wireless Bluetooth unit. The block diagram of the system software is presented in Fig. 3. The four parts of the GUI are:

- Communication controller: This part ensures a high speed wireless (Bluetooth) link between the $\mathrm{PC}$ and the main controller and processor unit. It periodically generates a "link test request" to verify the wireless channel.

- PGA controller: This part allows the user to select individual amplification ratios for each detector. An auto-range mode is also available, in which the system automatically adjusts amplification of the detected signal according to the light intensity actually detected. As this process amplifies both the signals and the noise, the SNR is not improved. Consequently, before applying 


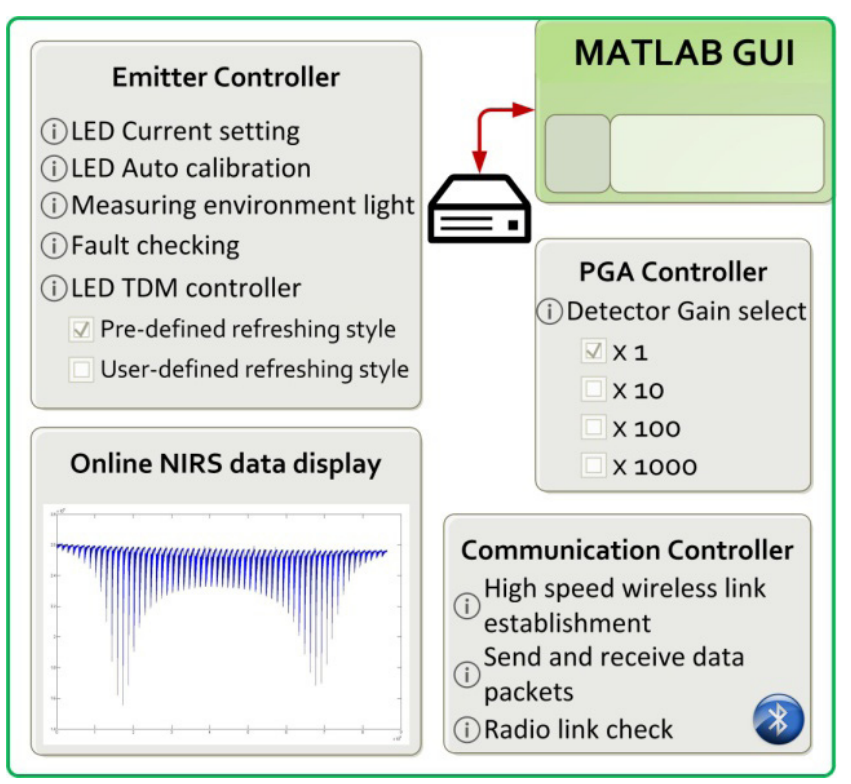

Fig. 3. WDA-NIRS software structure: A GUI provides access to three controllers for assisted manipulation of LEDs (emitter controller), detectors (PGA controller) and communication and data visualization (displayed data are derived from experiments with our "Sensitivity Scanner" phantom).

this step, the auto-calibration software tries to maintain a constant PGA gain of $\times 1$, and increases the signal level by adjusting the power of the emitted light. The signal level is therefore increased, while the background noise remains essentially unchanged, and the overall SNR is improved. When this correction is not sufficient, the gain of the detector can be increased in a stepwise fashion.

- Emitter controller: This part allows free choice of time multiplexing and current setting for the light sources. It contains a number of predefined standard settings, but can also be used to create customized user-defined formats. For this purpose, a special dual port RAM for refreshing information was included in the FPGA. A special state is designed for each light source and all states are periodically refreshed. Consequently, by applying the desired value to each state ("on", "off" or "all off" for measuring the environmental light), the user can define any required source multiplexing according to the specific application.

Furthermore, the power of the emitted light can be individually selected for each LED with 128-step resolution, or automatically determined by autocalibration, in which the emitter current is iteratively adapted with respect to the detector output signal. Therefore, all the LEDs are initially powered off and the environmental light is measured and displayed. The first LED is then set to half maximal output power and the back-scattered light is measured. This measurement is used to iteratively optimize the intensity in 7 steps ( 7 bit resolution).

This new feature allows fast and easy adjustment of light intensity in response to the multitude of different influences e.g., background light, related tissue type, skin color, etc. Settings as determined for each channel (LED-SPD combination) and the refreshing style protocol are registered in separate files. They are subsequently used to adjust the acquired signals before applying the modified Beer-Lambert law (MBLL). ${ }^{14}$

- Display and save module: This part offers two different modes, the first mode displays raw data and the second mode displays online preprocessed data. Appropriate algorithms have been developed and implemented to decompose the incoming data stream into time sequences for each channel and, in parallel, to store the related values for each PGA amplification and LED current. In addition, for each LED the changes in back-scattered light intensity for the two wavelengths are converted to changes in $\mathrm{HbO}$ and HbR concentration by applying the MBLL. Raw and preprocessed data are recorded in parallel for further offline processing.

\subsection{Wireless distributed signal acquisition}

The WDA-NIRS system presented here consists of a base station (portable PC) and an arbitrary number of acquisition modules. The base station provides the GUI to customize the desired acquisition parameters (including the number of modules in the distributed system and their structure) as well as programming, initialization, synchronization, failure detection/handling and data acquisition of all modules. The system provides programmable multiplexing parameters for each of the seven optional channels. Patches with a linear configuration, as presented in Figs. 1(c) and 1(d), are recommended for depth-resolving measurements. In the future, appropriate post-processing of the acquired signals will allow more precise extraction of the data of interest. When performing an experiment, the required number of acquisition modules and their 


\section{J. Safaie et al.}

corresponding patches are fixed at the measuring sites selected on the body. All modules are initialized by the PC station, synchronized signal acquisition is launched and data from all modules are recorded continuously by the PC-base while the subject is free to move within a distance of $100 \mathrm{~m}$ from the receiver.

\subsection{Phantom: Sensitivity scanner}

The scheme of our recently introduced experimental setup for sensitivity scanning ${ }^{14}$ is shown in Fig. 4(a). It consists of five different parts: (1) Control and data acquisition unit, (2) three-dimensional (3D) scanning unit, (3) optical tissue-like dynamic phantom, (4) patch, (5) NIRS main controller-processor board and (6) perturbation element.

The sensitivity scanner phantom consists of two major components, a background optical phantom and an optical perturbation. The background optical phantom is realized as a liquid contained in a transparent tank $\left(120 \times 120 \times 120 \mathrm{~mm}^{3}\right)$. The use of a liquid tissue phantom allows for free positioning and movement of the optical perturbation inside the phantom volume. In addition, a liquid phantom offers the possibility to change freely optical properties. Ingredients of the liquid phantom are distilled water, "Medialipide ${ }^{\circledR}$ (B.BRAUN Medical Inc) and "india-ink" (Lefranc \& Bourgeois ${ }^{\circledR}$ ). The concentration of Medialipide and india-ink is chosen to achieve various optical parameter values as needed for the experiments mimicking physiological conditions $\left(\mu_{a}=0.001 \mathrm{~mm}^{-1}\right.$ and $\left.\mu_{s}^{\prime}=0.8 \mathrm{~mm}^{-1}\right) .{ }^{14}$

To avoid any sedimentation effects, a magnetic stirrer is turning continuously throughout the whole experiment. The optical perturbation is realized by a liquid-filled glass bulb. This home-made glass bulb is thin walled $(\sim 0.2 \mathrm{~mm}$ thick $)$ and spherical shaped $(2 \mathrm{~mm}$ diameter). The liquid in the glass bulb can be freely chosen to meet the desired optical properties normally with respect to the liquid of the background. The glass bulb end is closed using transparent silica gel (glass adhesive). It is held in position using a L-shaped glass tube, which is filled with the same liquid as used for the background tissue phantom. By integrating a $100 \times 50 \mathrm{~mm}$ window in the tank wall covered by a $0.1 \mathrm{~mm}$ PVC transparent film, the refraction effects on patch emitters/detector due to transparent perspex walls (8 $\mathrm{mm}$ thickness) have been reduced.

The 3D scanning unit performs the computercontrolled movement of the perturbation through the region of interest of the phantom volume according to Fig. 4(b). For this purpose, the volume of interest is subdivided in a sequence of vertical parallel lines followed meander wise from the front to the back forming thus a page, which is followed by the next page in a distance of $1 \mathrm{~mm}$ beside. The distance between the scanning lines had been chosen to $1 \mathrm{~mm}$ as well. In the presented

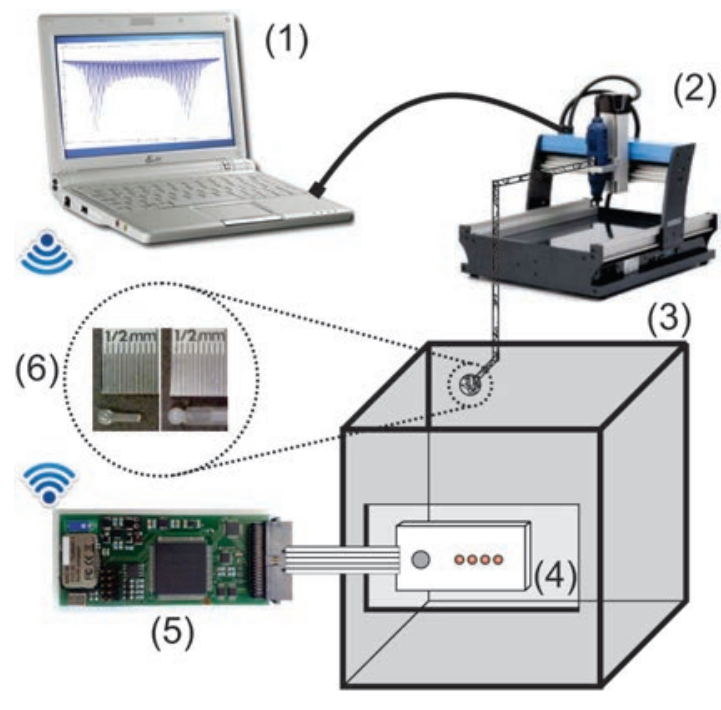

(a)

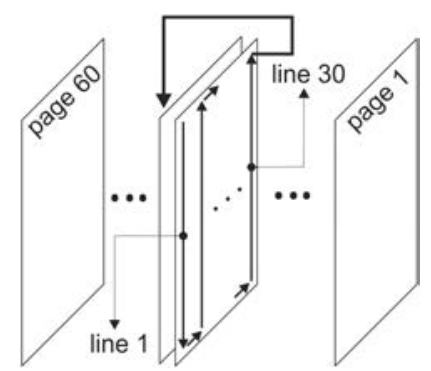

(b)

Fig. 4. Sensitivity scanner: (a) Experimental setup with (1) control and data acquisition unit, (2) 3D scanning unit, (3) optical tissue-like dynamic phantom, (4) patch, (5) NIRS main control and processing unit and (6) perturbation element. (b) The scanning is performed in 60 consecutive pages each of which consisting of 30 lines executed meander wise from the front to the back. 
experiments, every volume consists of 60 pages with 30 lines each.

All experiments have been carried out in a dark room to prevent from any signal perturbation by environment light. In addition, to compensate all kind of jeopardizing influences, as light piping by transparent films, the effects of glass, etc., we performed in any case two experiments one with the actual test medium in the probe and one with the probe filled with background medium. Then, the difference of the two acquired signals was further evaluated.

\subsection{Human subject studies}

\subsubsection{Validation with standard clinical cerebral oxygenation measuring system}

To verify the appropriate overall functioning of the WDA-NIRS system in human tissue measurements, we performed arterial occlusion experiments. The NIRO-200NX system was employed as the standard clinical system, since it is used widely for cerebral oxygenation measurements. The patches were attached at the forearm and a blood pressure cuff was fixed at the upper arm. The experiments have been done sequentially with the same protocol: after 1 min registration in relax state, the cuff was inflated to $250 \mathrm{mmHg}$ for $2 \mathrm{~min}$ to close arm arteries and veins and then the monitoring was continued for $3 \mathrm{~min}$.

\subsubsection{Application demonstrating the depth-resolving potential}

An experiment was conducted to demonstrate the need for and the feasibility of depth-resolving experiments and their importance for interpretation of the acquired data.

We first investigated the influence of a short period of apnea on $\mathrm{HbR}$ and $\mathrm{HbO}$ concentration in the frontal scalp and brain compared to the right forearm skin and pronator muscle. For this purpose, a depth-resolving linear patch with one detector and eight emitters was placed on the right forehead in a horizontal plane and over the right forearm pronator muscle. The subject was comfortably seated during the whole recording while executing the following protocol: $30 \mathrm{~s}$ of relaxation for baseline acquisition, $30 \mathrm{~s}$ of end-inspiratory breath-holding,
$60 \mathrm{~s}$ of relaxation for recovery. The 16 channels were normalized to the mean value obtained during the first $30 \mathrm{~s}$ of acquisition. The relative changes were therefore determined with respect to baseline, allowing direct comparison of concentration changes in various tissue layers. The signals were detrended and low-pass filtered $(0.8 \mathrm{~Hz}$ cut-off frequency). The differential pathlength factor (DPF) values for 760 and $850 \mathrm{~nm}$ wavelength have been determined as 6.2 and 5.3, respectively (as evaluated in Ref. 22).

\subsubsection{Application demonstrating distributed acquisition}

We conducted stair negotiation experiments to demonstrate the advantages of a wearable, distributed acquisition system for advanced clinical diagnosis.

In the simple example presented here, two units were used. One unit was attached over the medial head of the gastrocnemius muscle, the large posterior muscle of the calf that raises the heel and therefore extends the foot. This muscle therefore provides the propelling force for running and jumping and plays a major role in walking up and down stairs. ${ }^{23-25}$ The other unit was attached horizontally to the middle of the left side of the forehead, thereby acquiring information about the microcirculation of the skin/scalp via the short EDD, and information about the microcirculation of the outer frontal part of the cerebrum via larger EDDs. All patches were maintained in place by the elastic tubular net bandage described above.

The corresponding signals were acquired and registered while the subject executed the following protocol: $60 \mathrm{~s}$ of rest in the upright position for baseline acquisition, ascending 10 stairs (1 per s), $50 \mathrm{~s}$ of recovery, descending 10 stairs (1 per s), $50 \mathrm{~s}$ of recovery. The acquired channels were normalized to the mean value obtained during the first $60 \mathrm{~s}$ of acquisition.

\section{Results}

\subsection{Validation with a sensitivity mapping phantom}

The first objective validation of this new device consisted of signal acquisition from our sensitivity mapping phantom. A complete 3D scan was acquired 


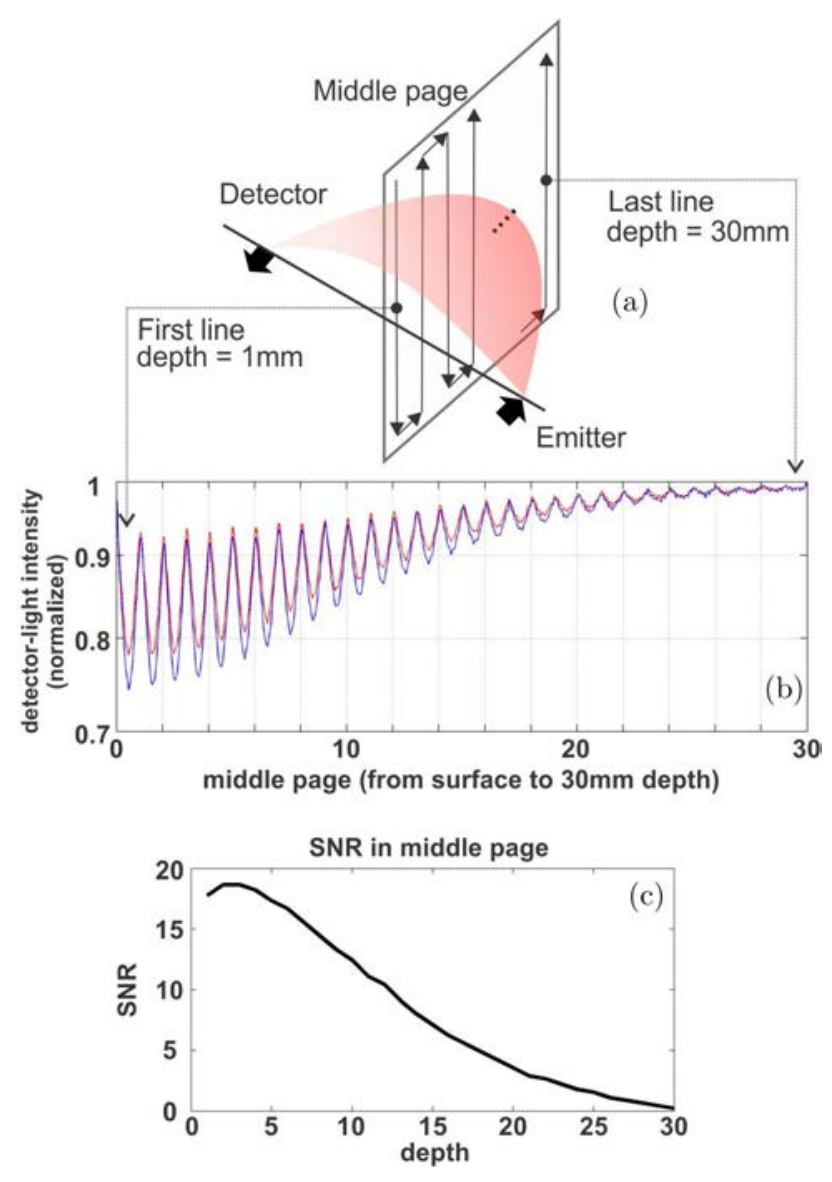

Fig. 5. Typical results of a phantom experiment. (a) Scanning of the middle page starting from the front layer back to the 30 th line at a depth of $30 \mathrm{~mm}$. (b) Signal acquired during the scan of the middle plane starting with the front line exhibiting a sequence of maxima from the middle positions and of minima from the top and bottom positions (blue/red curves for IR/Red lights, respectively). (c) SNR of the IR-signal as function of the depth determined for the middle page (b).

for the entire phantom in a dark room. Figure 5(a) shows the scanning protocol for the page in the middle between emitter and detector, which is the region of greatest interest in this context. The scanning starts from the most superficial layer back to the $30 \mathrm{th}$ line at a depth of $30 \mathrm{~mm}$. The signal acquired during this scan is presented in Fig. 5(b). It exhibits a sequence of peaks and minima corresponding to the sensitivity along the vertical scanning lines, with an interline distance of $1 \mathrm{~mm}$ [see Fig. 5(b)]. The 30 peaks therefore provide a measure for the sensitivity, as their amplitude decreases from $1 \mathrm{~mm}$ below the surface to a depth of $30 \mathrm{~mm}$. For SNR measurement, the background noise of the system was determined (i.e., from a measurement with the optical perturbation element positioned outside of the phantom) and the SNR for the middle-line of the middle page was then calculated [see Fig. 5(c)].

\subsection{Arterial occlusion experiment}

The results are presented in Fig. 6. In WDA-NIRS, two emitter-detector pairs are selected who had the same inter-optode distance as in the NIRO200NX system. The WDA-NIRS system calculates online changes in hemoglobin concentration by MBLL and the tissue oxygenation index (where TOI is defined by concentration of $\mathrm{HbO}$ divided by $\mathrm{Hb}_{\text {tot }}$ concentration ${ }^{9}$ ) based on the SRS method. ${ }^{9}$ After arterial occlusion the TOI reduced notably (from $67 \%$ to $55 \%$ ), increased immediately after opening and returned after an overshoot to the initial value within few minutes. The results of the two identical sequential experiments with both systems show good correlation.

\subsection{Apnea: Skin and deep tissue}

Experiments were carried out one to three times on five different subjects. The results are shown in Fig. 7. The time series for the first five LEDs situated at a distance of between 10 and $30 \mathrm{~mm}$ show the reaction of the microcirculation at the two acquisition sites. Marked differences in dynamics and magnitude of changes in HbR concentrations were observed between the forehead and the forearm and according to the depth of the tissue. The forearm response was generally higher in terms of $\mathrm{HbR}$ concentration.

Changes in the forehead skin (short EDDs) were always more pronounced than for longer EDDs, i.e., for signals also containing in addition information from deeper brain tissue. In contrast, in the forearm, changes in $\mathrm{HbR}$ and total hemoglobin $\left(\mathrm{Hb}_{\text {tot }}\right)$ concentration were less marked for the skin than for signals containing information from deeper tissue (muscle). The HbR changes observed in the brain were much smaller (one order of magnitude!) than for $\mathrm{Hb}_{\text {tot }}$ (corresponding to $\mathrm{HbO}$ ) concentration, which is in line with the results reported by MacIntosh et al. for similar experiments. ${ }^{26}$

\subsection{Stair negotiation}

The stair negotiation experiment was performed twice by one female and two male subjects. The results are shown in Fig. 8. Marked differences in changes in tissue hemoglobin concentration due to 

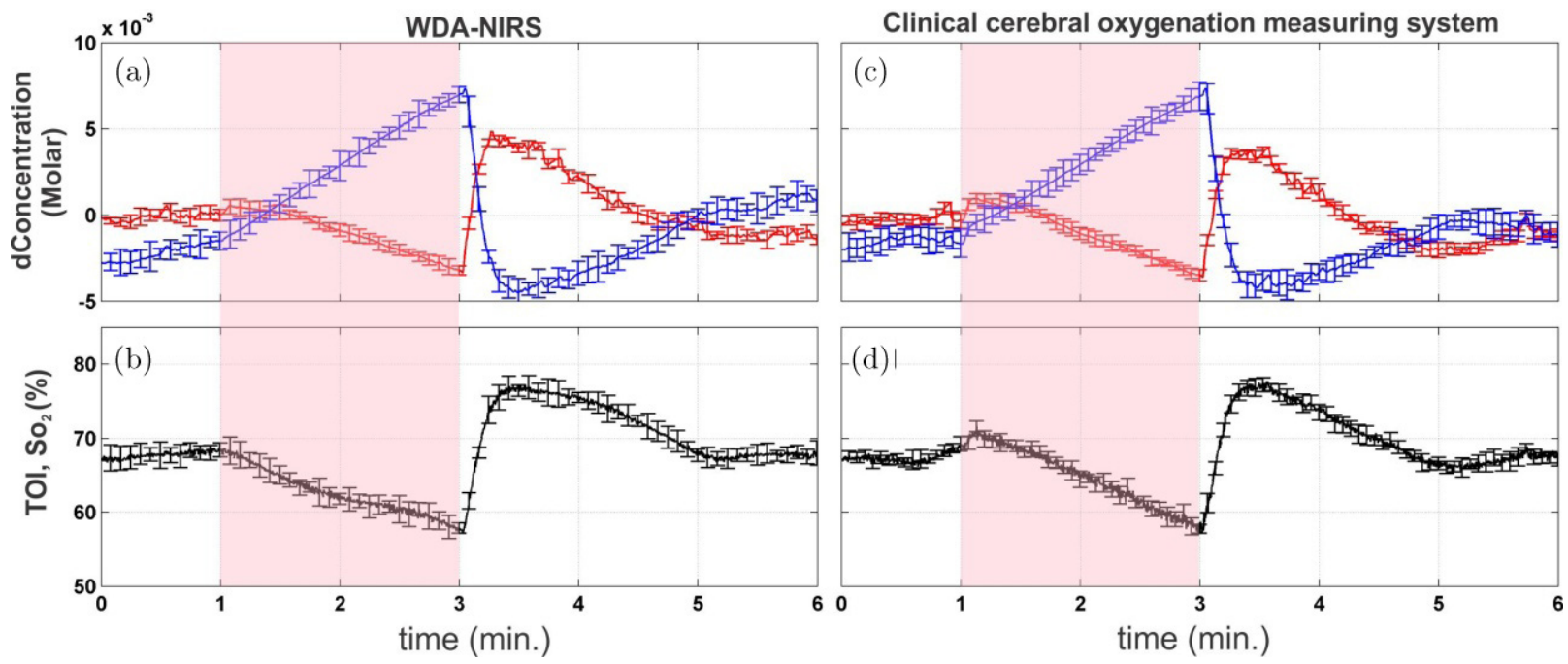

Fig. 6. Comparison of results from arterial occlusion experiments with our WDA-NIRS (left) and a NIRO-200NX clinical system (right). (a) and (c) represent $\mathrm{HbO}$ (red) and $\mathrm{HbR}$ (blue), while (b) and (d) show the arm TOI for WDA-NIRS and standard clinical system, respectively. The transparent pink areas represent the arterial and venous occlusion period.

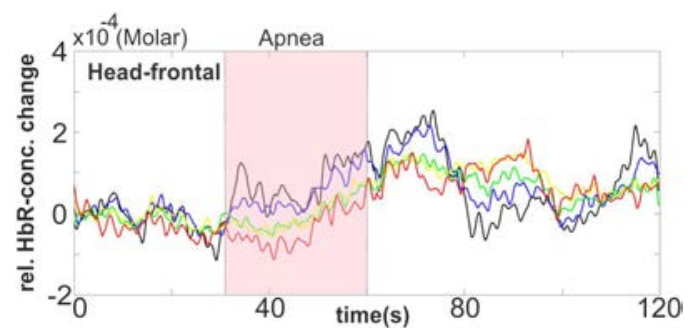

(a)

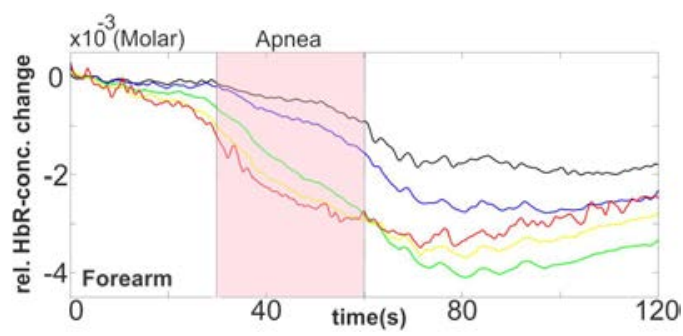

(c)

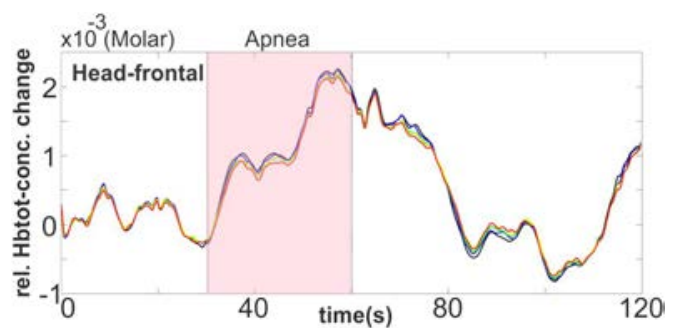

(b)

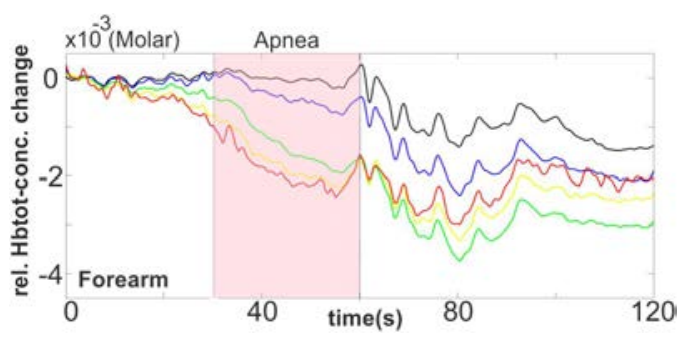

(d)

Fig. 7. Apnea (breath-holding between the 30th and 60th second) induced hemoglobin concentration changes. The upper line $(a, b)$ shows changes acquired from the forehead and the lower line $(c, d)$ shows changes acquired from the right forearm over the pronator muscle. Graphs on the left $(\mathrm{a}, \mathrm{c})$ show relative HbR concentration changes, and graphs on the right (b, d) show changes in $\mathrm{Hb}_{\text {tot }}$ concentration. The inter-optode distance is color-coded from the shortest to the longest distance: black $=1 \mathrm{~cm}$, blue $=1.5 \mathrm{~cm}$, green $=2 \mathrm{~cm}$, yellow $=2.5 \mathrm{~cm}$ and red $=3 \mathrm{~cm}$.

physical activity were observed at the two acquisition sites. While changes in the leg muscle were considerable and more or less parallel for $\mathrm{HbR}$ and $\mathrm{HbO}$ concentrations, the forehead recordings showed even more marked differences for $\mathrm{HbO}$ concentration, with less marked changes for HbR. These results can probably be attributed to the above-mentioned (over)compensation, which aims to keep the HbR concentration in the cerebrum at a constant low level. ${ }^{26}$ 


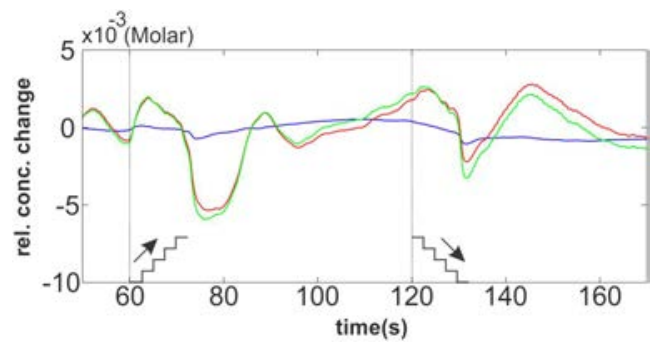

(a)

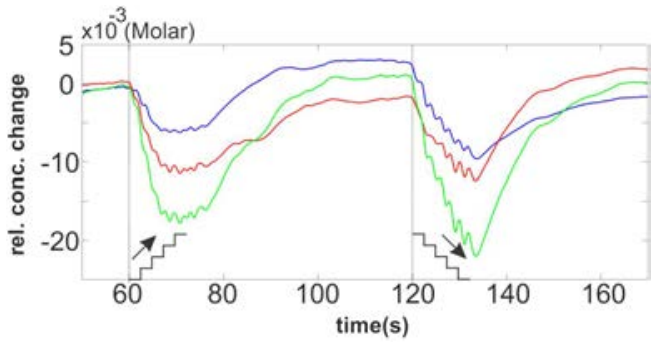

(b)

Fig. 8. Changes in hemoglobin concentration due to stair negotiation: (a) in the forebrain and (b) in the gastrocnemius muscle. Blue indicates $\mathrm{HbR}$, red indicates $\mathrm{HbO}$ and green indicates $\mathrm{Hb}_{\text {tot }}$. The time series was derived from the 4th LED (25 mm interoptode distance).

\section{Discussion and Conclusion}

Our flexible, wireless WDA-NIRS allows mobile, multi-site, highly accurate and safe distributed NIRS signal acquisition. It consists of up to seven distinct controller and acquisition modules, which can be freely positioned over the body. The signal acquisition units are each smaller than a cigarette box (weight of each unit with the battery $=48 \mathrm{~g}$ ) and synchronized by a nearby PC station. The acquisition units can be programmed individually to adapt them to particular local needs depending on the microcirculation of interest in different muscles, brain, skin, etc. The choice of a FPGA as the core of the control unit was pivotal to develop the advanced built-in features of this instrument, especially the inbuilt capacity for increased depth resolution in microcirculation analysis. The signals acquired by the system must therefore present adequate and sufficient quality for each channel under varying circumstances, which means that the power of the emitted light must be adjusted to the actual local conditions such as different EDDs, sensor position, skin color, environmental light, etc. The hardware/software provided therefore comprises a "light power auto-calibration" mode GUI, which can be used to program the light emitted by each LED, individually for each instant of the refresh cycle, with a 7 bit resolution (128 steps) in the current version. Since complete auto-calibration takes several seconds in the current version, this procedure is only applied during setup.

To enhance the inbuilt capacity of depth-resolving microcirculation analysis, we created an appropriate patch, composed of a sequence of 8 inline positioned dual wavelength emitters, allowing signal acquisition for EDDs ranging from 10 to $45 \mathrm{~mm}$ ( $45 \mathrm{~mm}$ is probably the longest distance that can be reasonably used with the technology described here).

\section{Application demonstrating experiments}

The stair negotiation experiment provides an example of the importance of distributed synchronized acquisition and portability. Stair negotiation constitutes one of the most difficult tasks attributable to aging and is one of the leading causes of fallrelated injuries. ${ }^{27}$ It is regularly used as a standard test, especially applied to evaluate performance in the elderly. Limited research has been conducted into the underlying factors that impair performance of this important activity of daily living. ${ }^{28,29} \mathrm{Ap}$ plication of our system as a diagnostic tool would obviously provide very useful additional information for the clinical differential diagnosis, as it simultaneously acquires information about the functioning of the musculoskeletal apparatus during movement and about related changes of the cerebral microcirculation.

The advantage of this approach is that the subject is required to execute a well-defined dynamic effort: elevating the body mass to a certain height in a certain time without any constraints to free movement. During this movement, detailed information concerning the microcirculation are acquired from distinct tissues situated at different depths.

The chosen combination of signal acquisition sites revealed a marked blood expulsion effect of gastrocnemius muscle contractions, as well as a marked drop in HbR concentration in brain tissue Fig. 8(a). This effect is probably related to incompletely compensated acceleration of the body. This more or less incomplete compensation might partly explain the problems encountered by elderly people 
in stair negotiation. These findings are consistent with those reported by Szufladowicz et al. for headup tilt table tests performed for evaluation of brain function during vasovagal syncope with NIRS. These authors found changes in $\mathrm{HbR}$ and $\mathrm{HbO}$ concentration in response to tilts similar to those reported here. ${ }^{30}$

\section{Depth-resolving potential}

The apnea experiments using the depth-resolving acquisition available with the hardware indicate the type of results that can be expected once appropriate depth-discriminating algorithms are available. For example, in Fig. 7(a) the signal indicates increase in $\mathrm{HbR}$ concentration in the outer tissue layer (black line), i.e., the scalp. This information makes also part of the red signal containing as well photons derived from deeper tissue i.e., cerebrum. With properly eliminating the information stemming from the outer surface layers (black signal) from the red signal, the resulting $\mathrm{HbR}$ concentration change will be less marked in the cerebrum, which would be consistent with the known compensatory increase of total blood flow in the brain during breath-holding ${ }^{26}$ as shown in Fig. 7(b). This demonstrates the need for acquisition of multidistance signals in NIRS and also the need for further algorithmic developments for reliable evaluation of these signals.

With the presented equipment, it will become possible to extract the information of interest for a particular tissue layer. Various methods have already been proposed for this type of signal decomposition. ${ }^{31-33}$ Further research is required to develop these approaches.

\section{General benefits}

These experiments highlight the potential benefits of diagnostic investigations in patients and normal subjects using the WDA-NIRS based on parallel and synchronized acquisition of valuable physiological and clinical information concerning the microcirculation under various conditions. The results show that the WDA-NIRS system is a reliable tool that can be used to acquire information that has been previously poorly accessible and that will be of interest in medical research and clinical diagnostics.

\section{Limitations and future improvements}

In the current version, complete auto-calibration for 16 light sources takes several seconds, which is too long for repeated controls during a running registration. In future versions, an online auto-control and correction mechanism will be integrated. This will be possible without hardware changes because of the in-system FPGA programming facilities.

It turned out that the actual 7 bit resolution $(42 \mathrm{~dB})$ is not sufficient to provide the complete power range of emitted light, which is needed for distances between 1 and $4.5 \mathrm{~cm}$. Only distances of up to $2.5 \mathrm{~cm}$ EDD can be evaluated. Therefore, the dynamic range of LED drivers must be increased to 12 bit, which is sufficient to cover the whole range needed for $3.5 \mathrm{~cm}$ differences.

Major efforts in terms of algorithm and software development are necessary to put into effect the depth-resolving potential of the instrument. It may therefore also be necessary to (re)design different types of emitter-detector configurations.

\section{Acknowledgments}

The financial support of the UPJV and Region of Picardie are gratefully acknowledged.

\section{References}

1. F. F. Jöbsis, J. H. Keizer, J. C. LaManna, M. Rosenthal, "Reflectance spectrophotometry of cytochrome aa3 in vivo," J. Appl. Physiol. 43(5), 858-872 (1977).

2. F. Wallois, M. Mahmoudzadeh, A. Patil, R. Grebe, "Usefulness of simultaneous EEG-NIRS recording in language studies," Brain Lang 121(2), 110-123 (2012).

3. M. Ferrari, V. Quaresima, "A brief review on the history of human functional near-infrared spectroscopy (fNIRS) development and fields of application," Neuroimage 63(2), 921-935 (2012).

4. G. M. Lech, "Using functional near-infrared spectroscopy to measure cognitive function: When will it become an accepted clinical tool for cognitive aging and prodromal dementia screening?" J. Innov. Opt. Health Sci. 4(04), 373-383 (2011).

5. R. V. Maikala, Y. N. Bhambhani, "Near-infrared monitoring of the lumbar erector spinae muscle in healthy men and women during static and dynamic endurance work," J. Innov. Opt. Health Sci. 4(02), 173-182 (2011).

6. A. C. Merzagora, M. T. Schultheis, B. Onaral, M. Izzetoglu, "Functional near-infrared spectroscopy-based assessment of attention impairments after traumatic brain injury," J. Innov. Opt. Health Sci. 4(03), 251-260 (2011). 
7. T. Hamaoka, K. K. McCully, "Muscle research work with britton chance from in vivo magnetic resonance spectroscopy to near-infrared spectroscopy," J. Innov. Opt. Health Sci. 4(03), 227-237 (2011).

8. Y. Hoshi, "Functional near-infrared spectroscopy: Potential and limitations in neuroimaging studies," Int. Rev. Neurobiol., 66, 237-266 (2005).

9. S. Suzuki, S. Takasaki, T. Ozaki, Y. Kobayashi, "A tissue oxygenation monitor using NIR spatially resolved spectroscopy," Proc. SPIE 3597, 582-592 (1999).

10. T. Muehlemann, D. Haensse, M. Wolf, "Wireless miniaturized in-vivo near infrared imaging," Opt. Express 16(14), 10323-10330 (2008).

11. B. R. Shadgan, "Wireless near-infrared spectroscopy of skeletal muscle oxygenation and hemodynamics during exercise and ischemia," Spectroscopy 23(5-6), 233-241 (2009).

12. G. Yurtsever, A. Bozkurt, F. Kepics, K. Pourrezaei, A. Devaraj, "Pocket PC based wireless continuous wave near infrared spectroscopy system for functional imaging of human brain," Proc. 25th Annual Int. Engineering in Medicine and Biology Society Conf. of the IEEE, Vol. 4, pp. 3435-3437 (2003).

13. Y. Zhang, J. W. Sun, G. Wei, F. Scopesi, G. Serra, P. Rolfe, "Design of a portable near infra-red spectroscopy system for tissue oxygenation measurement," Proc. 3rd Int. Conf. Bioinformatics and Biomedical Engineering ICBBE pp. 1-4 (2009).

14. A. V. Patil, J. Safaie, H. A. Moghaddam, F. Wallois, R. Grebe, "Experimental investigation of NIRS spatial sensitivity," Biomed. Opt. Express 2(6), 1478-1493 (2011).

15. S. R. Arridge, "Photon-measurement density functions. Part I: Analytical forms," Appl. Opt. 34(31), 7395-7409 (1995).

16. Y. Zhang, J. Sun, P. Rolfe, "Reduction of global interference in functional multidistance nearinfrared spectroscopy using empirical mode decomposition and recursive least squares: A Monte Carlo study," J. Eur. Opt. Soc. — Rapid Publications 6, 11033-11010 (2011).

17. C. J. Soraghan, T. E. Ward, F. Matthews, C. Markham, "Optical safety assessment of a nearinfrared brain-computer interface," Proc. IET Irish Signals and Systems Conf. ISSC, pp. 174-179 (2008).

18. A. Bozkurt, B. Onaral, "Safety assessment of near infrared light emitting diodes for diffuse optical measurements," Biomed. Eng. Online 3(1), 9 (2004).

19. G. Strangman, M. A. Franceschini, D. A. Boas, "Factors affecting the accuracy of near-infrared spectroscopy concentration calculations for focal changes in oxygenation parameters," Neuroimage 18(4), 865-879 (2003).

20. K. Uludag, J. Steinbrink, A. Villringer, H. Obrig, "Separability and cross talk: Optimizing dual wavelength combinations for near-infrared spectroscopy of the adult head," Neuroimage $\mathbf{2 2}(2)$, 583-589 (2004).

21. M. K. Wallin, S. Wajntraub, "Evaluation of Bluetooth as a replacement for cables in intensive care and surgery," Anesth. Analg. 98(3), 763-767 (2004).

22. A. Duncan, J. H. Meek, M. Clemence, C. E. Elwell, L. Tyszczuk, M. Cope et al., "Optical pathlength measurements on adult head, calf and forearm and the head of the newborn infant using phase resolved optical spectroscopy," Phys. Med. Biol. 40(2), 295-304 (1995).

23. T. P. Andriacchi, G. B. Andersson, R. W. Fermier, D. Stern, J. O. Galante, "A study of lower-limb mechanics during stair-climbing," J. Bone Joint Surg. Am. 62(5), 749-757 (1980).

24. A. Protopapadaki, W. I. Drechsler, M. C. Cramp, F. J. Coutts, O. M. Scott, "Hip, knee, ankle kinematics and kinetics during stair ascent and descent in healthy young individuals," Clin. Biomech. (Bristol, Avon) 22(2), 203-210 (2007).

25. S. Nadeau, B. J. McFadyen, F. Malouin, "Frontal and sagittal plane analyses of the stair climbing task in healthy adults aged over 40 years: What are the challenges compared to level walking?" Clin. Biomech. (Bristol, Avon) 18(10), 950-959 (2003).

26. B. J. MacIntosh, L. M. Klassen, R. S. Menon, "Transient hemodynamics during a breath hold challenge in a two part functional imaging study with simultaneous near-infrared spectroscopy in adult humans," Neuroimage 20(2), 1246-1252 (2003).

27. J. K. Startzell, D. A. Owens, L. M. Mulfinger, P. R. Cavanagh, "Stair negotiation in older people: A review," J. Am. Geriatr. Soc. 48(5), 567-580 (2000).

28. A. C. Tiedemann, C. Sherrington, S. R. Lord, "Physical and psychological factors associated with stair negotiation performance in older people," $J$. Gerontol. A Biol. Sci. Med. Sci. 62(11), 1259-1265 (2007).

29. A. A. Butler, J. C. Menant, A. C. Tiedemann, S. R. Lord, "Age and gender differences in seven tests of functional mobility," J. Neuroeng. Rehabil. 6, 31 (2009).

30. E. Szufladowicz, R. Maniewski, E. Kozluk, A. Zbiec, A. Nosek, F. Walczak, "Near-infrared spectroscopy in evaluation of cerebral oxygenation during vasovagal syncope," Physiol. Meas. 25(4), 823-836 (2004).

31. Y. Zhang, D. H. Brooks, M. A. Franceschini, D. A. Boas, "Eigenvector-based spatial filtering for 
reduction of physiological interference in diffuse optical imaging," J. Biomed. Opt. 10(1), 11014 (2005).

32. S. G. Diamond, T. J. Huppert, V. Kolehmainen, M. A. Franceschini, J. P. Kaipio, S. R. Arridge, et al. "Physiological system identification with the Kalman filter in diffuse optical tomography," Med. Image
Comput. Comput. Assist. Interv. 8(Pt 2), 649-656 (2005).

33. K. E. Jang, S. Tak, J. Jung, J. Jang, Y. Jeong, J. C. Ye, "Wavelet minimum description length detrending for near-infrared spectroscopy," J. Biomed. Opt., 14(3), 034004 (2009). 